\title{
Evaluation of pressure in a plasma produced by laser ablation of steel
}

\author{
Jörg Hermann ${ }^{\mathrm{a}, *}$, Emanuel Axente ${ }^{\mathrm{b}}$, Valentin Craciun ${ }^{\mathrm{b}}$, Aya Taleb ${ }^{\mathrm{a}, \mathrm{c}}$, Frédéric Pelascini ${ }^{\mathrm{c}}$ \\ ${ }^{a}$ Aix-Marseille University, CNRS, LP3, 13009 Marseille, France \\ ${ }^{b}$ National Institute for Lasers, Plasma and Radiation Physics, 77125 Măgurele, Romania \\ ${ }^{c}$ CRITT Matériaux Alsace, 67305 Schiltigheim, France
}

\begin{abstract}
We investigated the time evolution of pressure in the plume generated by laser ablation with ultraviolet nanosecond laser pulses in a near-atmospheric argon atmosphere. These conditions were previously identified to produce a plasma of properties that facilitate accurate spectroscopic diagnostics. Using steel as sample material, the present investigations benefit from the large number of reliable spectroscopic data available for iron. Recording time-resolved emission spectra with an echelle spectrometer, we were able to perform accurate measurements of electron density and temperature over a time interval from $200 \mathrm{~ns}$ to $12 \mu \mathrm{s}$. Assuming local thermodynamic equilibrium, we computed the plasma composition within the ablated vapor material and the corresponding kinetic pressure. The time evolution of plume pressure is shown to reach a minimum value below the pressure of the background gas. This indicates that the process of vapor-gas interdiffusion has a negligible influence on the plume expansion dynamics in the considered timescale. Moreover, the results promote the plasma pressure as a control parameter in calibration-free laser-induced breakdown spectroscopy.
\end{abstract}

Keywords: Kinetic pressure; Particle diffusion; Plasma modeling; Spectra simulation; Calibration-free LIBS.

\section{Introduction}

In pulsed laser ablation under conditions typically applied during material analyses via laser-induced breakdown spectroscopy (LIBS), the plasma generated at the sample surface is characterized by a temperature of several $\mathrm{eV}$ and a density close to solid density during the initial stage of expansion [1]. The plume undergoes a rapid explosion-like expansion until it reaches a pressure equilibrium with the surrounding compressed gas. On a longer timescale, the processes of heat and particle diffusion take place and contribute to the further evolution of plasma properties [2]. The precise scenario of the LIBS plasma development depends naturally on the laser irradiation conditions and the gas environment. Compared to short pulse laser excitation, nanosecond laser ablation takes advantage from the interaction of the laser beam with the vaporized material. This process changes the thermodynamic pathway of the laser-heated matter and leads to larger degrees of atomization and excitation [3]. In the case of ultraviolet (UV) radiation, laser heating occurs exclusively in

*Corresponding author: hermann@lp3.univ-mrs.fr a tiny volume close to the sample surface where the plasma density is high enough. This leads to an increased laser energy coupling towards the sample for the short-wavelength radiation. Plasma screening via laser energy absorption by the surrounding atmosphere is negligible and the background gas is heated by the shock wave and the interaction with the ablated vapor plume only [4].

The background gas has a strong influence on the properties of the laser-produced vapor plasma. In a molecular gas, such as ambient air, inelastic collisions play a significant role in the vapor-gas interaction. The energy loss at the vapor-gas contact front leads to the formation of a cold peripheral layer within the vaporized material [5]. The situation is different for ablation in an inert gas. Because of the large excitation energy, the plasma electrons interact with the background gas atoms mainly through elastic collisions, conserving thus their energy. This suppresses the formation of the cold border within the ablated vapor plume. The plasma appears uniform and diagnostics are easy to handle as they do not require space-resolved measurements. If furthermore the assumption of local thermodynamic equilibrium (LTE) is valid, the laser-produced plasma 
can be considered as an "ideal" radiation source for which the emission spectrum is accurately described by the simple model of a uniform LTE plasma [6].

In the present paper, we explore the unique properties of the ideal radiation source to ascertain an aspect of crucial interest for the understanding of LIBS fundamentals: the pressure-evolution within the laser-produced vapor plasma that is correlated to the expansion dynamics and the vapor-gas interdiffusion process. In fact, many authors reported diagnostics of LIBS plasmas with values of temperature $T$ and electron number density $n_{e}$ that indicate a pressure below the ambient gas pressure for a time of several microseconds after the laser pulse. For example, Gornushkin and Kazakov [7] reported $n_{e}=10^{16} \mathrm{~cm}^{-3}$ and $T=8000 \mathrm{~K}$ for a delay of several microseconds during laser ablation of aluminum in air. Assuming local thermodynamic equilibrium, the vapor pressure would be of more than one order of magnitude below the ambient pressure, if no vapor-gas mixing occurred. The authors concluded that rapid diffusion of air into the vapor plume ensured the pressure equilibrium at $P \cong 1 \mathrm{~atm}$. This explanation seems to be in contradiction to the expansion dynamics of LIBS plasmas predicted by computer-simulations that indicate a welldefined vapor-gas contact front for the investigated time interval until $1 \mu \mathrm{s}$ [8]. Similar values of electron density and temperature were reported for delays of some microseconds under different experimental conditions $[9,10,11,12]$. The existence of equilibrium is often discussed, but the questions of pressure and/or vapor-mass interdiffusion were mostly ignored [13].

Because of the high complexity of the involved mechanisms, modeling of nanosecond laser ablation into a background gas at atmospheric or near-atmospheric pressure is challenging. Calculations being based on simplifying assumptions, they enable only a qualitative description that is helpful in the identification and understanding of the phenomena [1]. Most computer simulations were performed for laser ablation in vacuum or low-pressure gas environment [14]. Calculations for ablation in air or other background gases at atmospheric pressure are mostly limited to the early stage of expansion where the pressure within the vapor plume still exceeds the ambient background gas pressure $[15,16,8]$. As an exception, Ershov-Pavlov et al. [17] calculated the expansion dynamics during Nd:YAG laser ablation of aluminum in air on a timescale up to $10 \mu \mathrm{s}$. The self-consistent calculations based on a two-dimensional Euler hydrodynamic model show that the pressure within the vapor plasma reaches a minimum for a delay of several microseconds, and that the minimum pressure is significantly lower than the ambient gas pressure.

The aim of the present work is to contribute to a better understanding of the later expansion stage of the LIBS plasma via the evaluation of the plasma pressure through accurate plasma diagnostics. Using steel as sample material, the present investigations take advantage of (i) reliable spectroscopic data that enable accurate measurements of plasma temperature and electron density, and (ii) the atomic structure of $\mathrm{Fe}$ and $\mathrm{Fe}^{+}$species that favor the establishment of local thermodynamic equilibrium.

\section{Experiment}

The plasma was generated by UV (266 nm) laser pulses of $4 \mathrm{~ns}$ duration from a frequency-quadrupled Nd:YAG laser source (Quantel, model Brilliant). The pulse energy was attenuated to $6 \mathrm{~mJ}$ by turning the beam polarization with a half-wave plate and crossing through a polarization analyzer. Focusing the laser beam with a lens of $150 \mathrm{~mm}$ focal length to a spot diameter of $100 \mu \mathrm{m}$, a laser fluence of about $80 \mathrm{~J} \mathrm{~cm}^{-2}$ was incident on the sample surface. The stainless steel sample (see Table 1) was placed on a motorized sample holder in a vacuum chamber of $10^{-4} \mathrm{~Pa}$ residual pressure. During the experiments, the chamber was filled with argon at $5 \times 10^{4} \mathrm{~Pa}$ pressure.

Optical emission spectroscopic measurements were performed by imaging the plasma with two lenses of 150 and $35 \mathrm{~mm}$ focal lengths onto the entrance of an optical fiber of $600 \mu \mathrm{m}$ core diameter. According to the image magnification 1:5, the entire plasma volume was observed. The fiber was coupled to an echelle spectrometer of $0.4 \mathrm{~m}$ focal length with two optical paths for the UV and visible/near infrared spectral ranges (LTB, model Aryelle Butterfly). Using an intensified charge-coupled device (ICCD) matrix detector for

Table 1: Composition of steel sample: mass fractions measured via inductively coupled plasma atomic emission spectroscopy $C_{I C P}$ and values deduced from the spectra analysis $C_{L I B S}$.

\begin{tabular}{ccc}
\hline Element & $C_{I C P}(\%)$ & $C_{\text {LIBS }}(\%)$ \\
\hline $\mathrm{Fe}$ & 70.0 & 69.5 \\
$\mathrm{Cr}$ & 18.4 & 18.8 \\
$\mathrm{Ni}$ & 9.5 & 9.7 \\
$\mathrm{Mn}$ & 0.94 & 0.94 \\
$\mathrm{Si}$ & 0.67 & 0.67 \\
$\mathrm{Ti}$ & 0.46 & 0.46 \\
\hline
\end{tabular}


photon detection, a resolving power of $1.4 \times 10^{4}$ and $9.4 \times 10^{3}$ is obtained for the UV and visible spectral ranges, respectively.

The spectral resolution of the apparatus was measured as a function of wavelength using a low-pressure argon-mercury lamp. An intensity calibration of the spectroscopic apparatus was performed in the visible and UV spectral ranges using calibrated tungsten and deuterium lamps, respectively.

The spectra were recorded for different delays of the detector gate $t_{g}$ with respect to the laser pulse, setting the gate width $\Delta t_{g}$ small enough so that the variations of temperature and electron density during the time of observation were small compared to their absolute values. Typically we applied $\Delta t_{g} \leq t_{g} / 2$. To enhance the signal-to-noise ratio, data acquisition was performed by averaging over 200 ablation events, applying 10 pulses on 20 different irradiation sites. The sites were separated by a distance of $150 \mu \mathrm{m}$.

Fast imaging of the expanding plume was performed with a focusing objective coupled to an ICCD detector. Each image was acquired by accumulating the signal over 5 ablation events on a single irradiation site.

\section{Calculation details}

\subsection{Simulation of emission spectrum}

We have recently shown [6] that the plasma produced by UV nanosecond laser ablation in near-atmospheric argon can be considered as an "ideal" radiation source as it combines two properties that are usually not found together: the plasma is uniform and in local thermodynamic equilibrium. In this case, the spectral radiance can be calculated by [18]

$$
B_{\lambda}=U_{\lambda}\left(1-e^{-\tau}\right),
$$

where $U_{\lambda}$ is the blackbody spectral radiance and $\tau$ the optical thickness. The optical thickness is given by $\tau=\int \alpha(z) d z=\alpha L$, where $\alpha$ and $L$ are the absorption coefficient and the plasma size along the line of sight, respectively. Considering all relevant transitions between bound and free excitation levels, the absorption coefficient writes

$$
\alpha(\lambda)=\sum_{i} \alpha_{\text {line }}^{(i)}(\lambda)+\alpha_{\text {ion }}(\lambda)+\alpha_{I B}(\lambda)
$$

where the sum includes the absorption coefficients $\alpha_{\text {line }}^{(i)}$ of transitions between bound levels. The terms $\alpha_{\text {ion }}(\lambda)$ and $\alpha_{I B}(\lambda)$ account for absorption through radiative ionization and inverse bremsstrahlung, respectively. The absorption coefficient of transitions between bound levels is given by [19]

$$
\alpha_{\text {line }}(\lambda)=\pi r_{0} \lambda^{2} f_{l u} n_{l} P(\lambda)\left(1-e^{-h c / \lambda k T}\right),
$$

where $r_{0}$ is the classical electron radius, $h$ is the Planck constant, $c$ is the vacuum light velocity, $k$ is the Boltzmann constant, $f_{l u}$ and $n_{l}$ are the absorption oscillator strength and the lower level population number density of the transition, respectively.

\subsection{Spectral line profile}

The normalized line profile $P(\lambda)$ is calculated considering Doppler and Stark broadening that are the dominant mechanisms of spectral line broadening in strongly ionized laser-produced plasmas [20]. Depending on the relative values of Doppler and Stark widths, the line shapes are described by Gaussian, Lorentzian or Voigt profiles. The Doppler width is calculated according to plasma temperature and atomic mass. The Stark width of each spectral line is obtained using Stark broadening parameters $w$ and assuming a linear dependence of the Stark width with the electron density [21]. For $n_{e}$-measurements via Stark broadening of the Fe I $538.33 \mathrm{~nm}$ transition, the temperature dependence of the Stark width was taken into account using [22]

$$
\Delta \lambda_{\text {Stark }}=w \frac{n_{e}}{n_{e}^{r e f}}\left(\frac{T}{T^{r e f}}\right)^{m} .
$$

Here, $n_{e}^{r e f}$ and $T^{r e f}$ are the reference values of $n_{e}$ and $T$ for which $w$ is tabulated (Appendix A, Table A.3). For the transitions other than Fe I $538.33 \mathrm{~nm}$, the $T$-dependence was unknown and therefore ignored. The laser-produced plasma being characterized by a strong variation of $n_{e}$ and a moderate variation of $T$, the disregard of the $T$-dependence of $\Delta \lambda_{\text {Stark }}$ is expected to have a small influence on the calculated spectral line profiles $[21,12]$. Since the Stark broadening parameters were not known for most transitions, we measured them in a separate experiment $[23,11]$. The Stark shift is obtained from Eq. (4) replacing $w$ by the Stark shift parameter.

\subsection{Plasma composition}

For the calculation of the plasma composition in local thermodynamic equilibrium, we refer to previous work $[24,25]$. Once the number densities of all plasma 


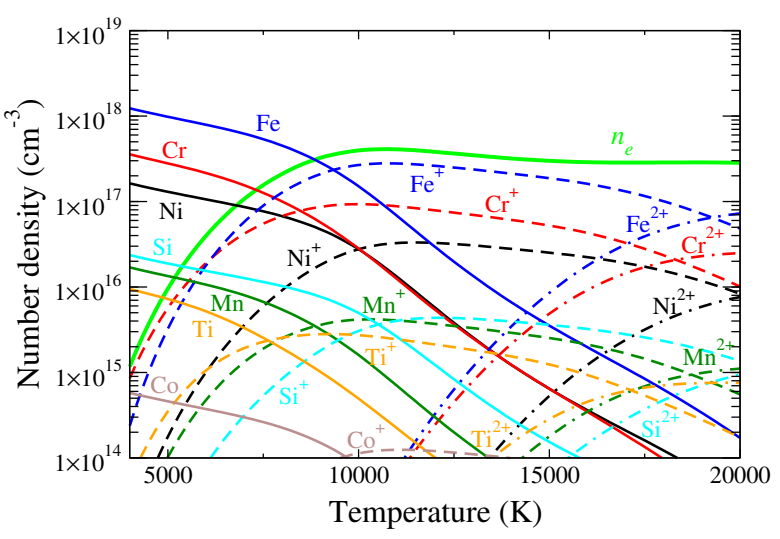

Figure 1: Number densities of species vs temperature computed for a plasma in LTE at atmospheric pressure with the elemental composition of the steel sample (see Table 1).

species are known, we compute the kinetic pressure using

$$
P=\left(n_{e}+\sum_{A} \sum_{z=0}^{z_{\max }} n_{A}^{z}\right) k T-\Delta P .
$$

Here, $n_{A}^{z}$ are the number densities of species from element $A$ with charge $z, \Delta P$ is the Debye pressure correction that accounts for the potential energy of charged species in the plasma. The sum includes all atomic species until a maximum charge $z_{\max }$. Molecular species are ignored as their abundance is negligible in the considered temperature range [14].

The number densities of the most abundant species are presented in Fig. 1 as functions of temperature. The calculations were performed assuming a plasma at atmospheric pressure. It is shown that the number densities of neutral atoms undergo a monotonic decrease. The densities of singly charged ions increase until $T \approx 10000 \mathrm{~K}$. For $T>10000 \mathrm{~K}$ the ionic species dominate the plasma composition but their number densities decrease to satisfy the pressure condition. Doubly charged ions have negligible number denisites for $T<15000 \mathrm{~K}$. They dominate the plasma composition for $T>20000 \mathrm{~K}$

For practical applications, Eq. 5 can be written as

$$
P=n_{e}\left(1+\frac{1}{\delta_{\text {ion }}}\right) k T-\Delta P,
$$

where $\delta_{i o n}$ is the ionization degree of the plasma. Thus, an approximate evaluation of pressure is possible from the measurements of $n_{e}$ and $T$ and the estimation of the ionization degree. Neglecting the Debye pressure correction, we obtain for a completely ionized plasma $\left(\delta_{\text {ion }}=1\right)$ the simplified expression $P \cong 2 n_{e} k T$.

\subsection{Plasma size along the line of sight}

The plasma size along the line of sight can be deduced from the intensity ratio of optically thin and thick transitions. In the optically thin case $(\tau<<1)$, Eq. (1) becomes

$$
B_{\lambda}^{\text {thin }}=\varepsilon_{\lambda} L,
$$

where the emission coefficient $\varepsilon_{\lambda}$ is related to the absorption coefficient via Kirchhoff's law of thermal radiation $\varepsilon_{\lambda} / \alpha=U_{\lambda}$. In the opposite case of large optical thickness $(\tau>>1)$, Eq. (1) simplifies to

$$
B_{\lambda}^{\text {thick }}=U_{\lambda} \text {. }
$$

The spectral radiance becomes independent of the plasma size and depends on temperature only. The plasma size $L$ can be deduced from the ratio $B_{\lambda}^{\text {thin }} / B_{\lambda}^{\text {thick }}$ of appropriate spectral lines. In practice, we first adjust the intensity of the measured spectrum to the computed radiance of the optically thin line. Then, we vary the plasma size until the computed radiance of the optically thick line matches the measured intensity.

\subsection{Calculation loop and line selection}

To compare the measured spectrum to the spectral radiance computed according to Eq. (1), $B_{\lambda}$ was convoluted with the apparatus spectral profile. Moreover, instead of calculating the absorption coefficients due to ionization and inverse bremsstrahlung [see Eq. (2)], the amount $\alpha_{i o n}(\lambda)+\alpha_{I B}(\lambda)$ was adjusted so that the intensity of computed continuum radiation equals the measured continuum intensity.

The spectral radiance of a uniform plasma in local thermodynamic equilibrium [Eq. (1)] depends on $n+2$ parameters: $T, n_{e}, L$ and the $n-1$ fractions of elements. We deduce all parameters applying the iterative measurement algorithm presented previously [25]. In the present work, the iterative loop includes an additional procedure of line selection according to their optical thickness and their interference with other transitions. Thus, lines with $\tau>1$ are excluded from the measurements of $T, n_{e}$, and elemental fractions.

An additional criterion applies to the selection of lines used for electron density measurements: their Stark width must be large enough to enable accurate $n_{e}$-measurements. 


\section{Results and discussion}

\subsection{Plasma diagnostics}

\subsubsection{Electron density measurement}

The measured and computed profiles of the transitions used for $n_{e}$-measurements are displayed in Fig. 2 for different recording times. According to the relative large Stark width (Appendix A, Table A.3), the Fe I $538.33 \mathrm{~nm}$ transition (a) enables accurate electron density measurements for $t>2 \mu \mathrm{s}$, when $n_{e}<10^{17} \mathrm{~cm}^{-3}$. For this transition, reliable Stark broadening parameters are available in literature for various values of plasma temperature [26]. We computed the Stark width using Eq. (4) with the values of $w$ and $m$ taken from Zielinski et al. [22]. The validity of $n_{e}$-measurements is proved by the linear correlation between measured Stark shift and Stark width shown in the inset of Fig. 2(a). The experiments being performed in a temperature-stabilized laboratory environment,
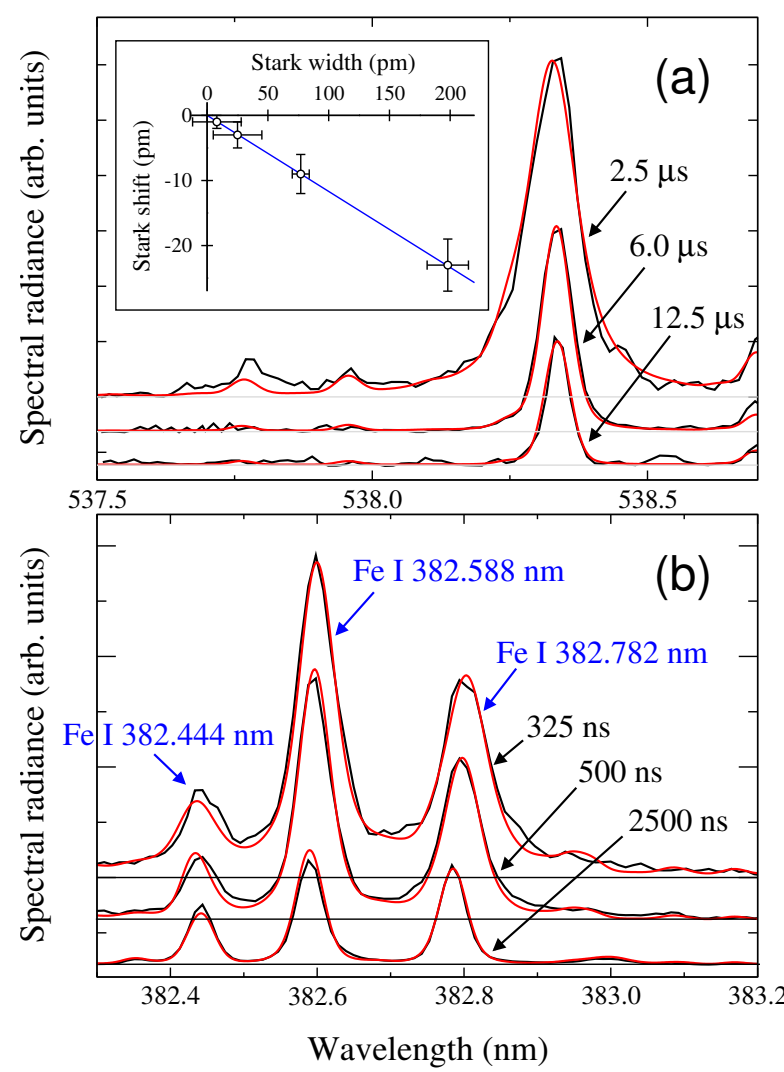

Figure 2: Measured (black lines) and computed (red lines) spectral radiances in the ranges selected for $n_{e}$-measurements. The electron density was deduced from Stark broadening of Fe I $538.33 \mathrm{~nm}$ and Fe I $382.78 \mathrm{~nm}$ for $t \geq 2500 \mathrm{~ns}$ (a) and $t \leq 2500 \mathrm{~ns}$ (b), respectively. The linear correlation between Stark width and shift proves the high accuracy of $n_{e}$-measurements (see insert)

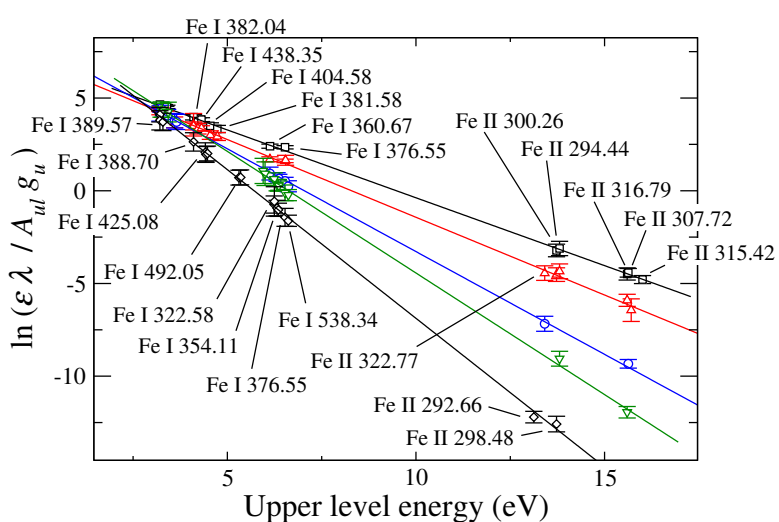

Figure 3: Saha-Boltzmann plot of $\mathrm{Fe}$ and $\mathrm{Fe}^{+}$transitions for $t=0.23 \mu$ s (squares), $0.33 \mu$ s (triangles up), $1.25 \mu \mathrm{s}$ (circles), $2.5 \mu \mathrm{s}$ (triangles down), and $6.0 \mu \mathrm{s}$ (diamonds) .

the shift measurements are of particular interest as they improve the $n_{e}$-measurement accuracy in the low electron density range (see error bars in the insert).

At early times, the strongly broadened Fe I $538.33 \mathrm{~nm}$ transition interfers with other transitions and the width measurement becomes inaccurate. Thus, the electron density is deduced for $t<2 \mu \mathrm{s}$ from Stark broadening of the Fe I $382.78 \mathrm{~nm}$ transition (b). This transition has a relatively large Stark shift (see Appendix A, Table A.2) that was explored to reduce the $n_{e}$-measurement uncertainty.

\subsubsection{Temperature measurement}

The temperature was deduced from the intensity ratio of spectral lines from atoms and ions as illustrated by the Saha-Boltzmann plot in Fig. 3. Here, the emission coefficient of each line is deduced from the measured spectrum using $\varepsilon=\varepsilon_{\text {comp }} B_{\text {meas }} / B_{\text {comp }}$, where $B_{\text {meas }}$ and $B_{\text {comp }}$ are the measured and computed line-integrated spectral radiances, respectively. The accurate description of spectral lines from both atoms and ions by a unique slope supports the hypothesis of a spatially uniform plasma. As a consequence of the variations of optical thickness and line interferences, the lines selected for $T$-measurements depend on the recording time. Typically, transitions of small Stark broadening parameter are used at early times, whereas strongly broadened lines are selected in priority at large delays.

\subsubsection{Time evolution of $n_{e}$ and $T$}

The electron density and plasma temperature are displayed in Fig. 4 as functions of time. To characterize 


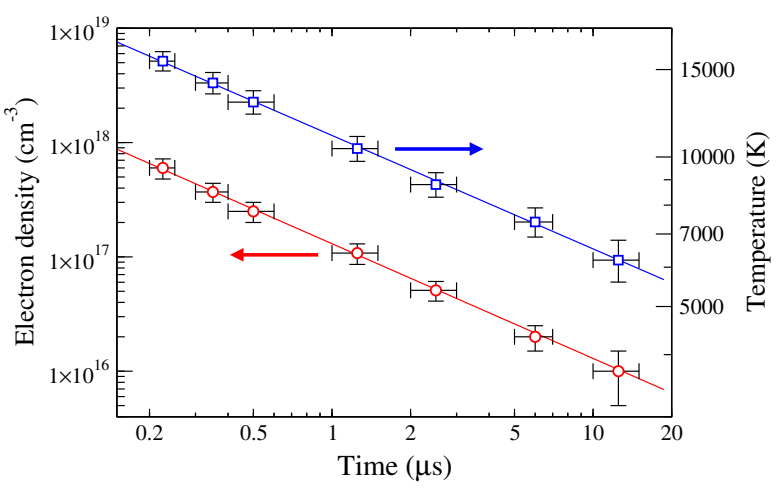

Figure 4: Electron density (circles) and temperature (squares) vs time. The vertical error bars represent the measurement uncertainties, whereas the horizontal error bars indicate the time interval of observation (gate width).

the decrease of $T$ and $n_{e}$, we approximate the measured data by functions $n_{e}, T=A t^{B}$ (continuous lines) with the parameters $A$ and $B$ deduced from the best fit. We obtain $n_{e}(t)=1.49 \times 10^{20} t^{-1.02}$ and $T(t)=$ $5.41 \times 10^{4} t^{-0.23}$, where $t, n_{e}$ and $T$ are in units of ns, $\mathrm{cm}^{-3}$ and $\mathrm{K}$, respectively. Combining both expressions, we obtain the electron density as a function of temperature $n_{e}(T)=0.152 T^{4.435}$.

\subsection{Characterization of pressure evolution}

\subsubsection{Determination of kinetic pressure}

The expression $n_{e}=f(T)$ deduced from the measurements presented in Fig. 4 is now used as a boundary condition for the calculation of the LTE plasma composition. Compared to the observations at constant atmospheric pressure (see Fig. 1) significant changes occur in the temperature dependence of plasma species when the $n_{e}=f(T)$-condition is applied (see Fig. 5). In opposition to the monotonic decrease at constant pressure, the temperature evolution of number densities of neutral atoms exhibits a minimum for $T \approx 12000 \mathrm{~K}$. Moreover, the number densities of doubly charged ions remain moderate, even for the largest considered temperature value. Both observations are supported by the experiment. Indeed, strong transitions from neutral atoms are detected since the early expansion stage over the entire time of observation. Contrarily, lines of doubly charged ions are rarely observed in the emission spectra recorded under experimental conditions typically applied in LIBS analyses [27].

In addition to the number densities of plasma species, the calculated kinetic pressure [Eq. (5)] is displayed in Fig. 5. The pressure is shown to reach a minimum value for a temperature of about $7000 \mathrm{~K}$. The pressure minimum is the consequence of the explosion-like expansion

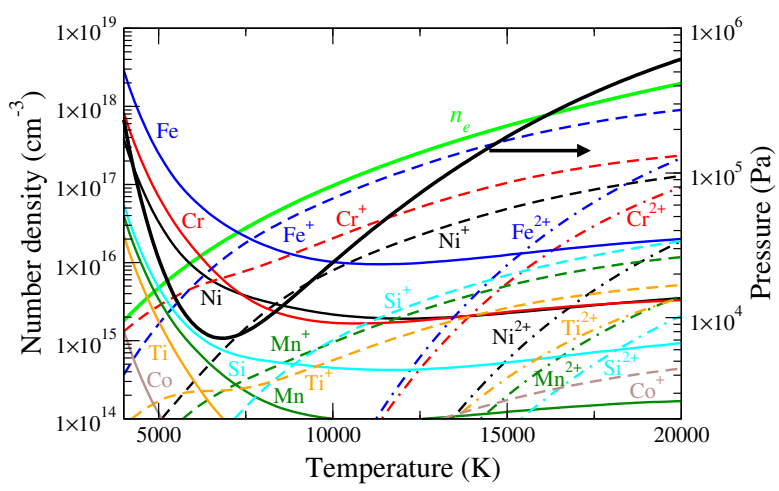

Figure 5: Number densities of species vs temperature computed for a plasma in LTE with the elemental composition of the steel sample (see Table 1). The calculations were performed for the $n_{e}=f(T)$ dependence deduced from the measurements (see Sec. 4.1.3). The pressure computed according to Eq. (5) is presented with respect to the secondary $y$-axis.

dynamics of the laser-produced plasma. Initially characterized by large values of density and temperature, the plume rapidly expands until the internal pressure equals the pressure of the surrounding compressed gas. As a result of further cooling due to energy loss via radiation, the pressure decreases to a minimum value below the ambient gas pressure.

\subsubsection{Pressure evolution and plasma expansion}

Inserting the measured time evolution of temperature (see Fig. 4) into the $P=f(T)$-dependence displayed in Fig. 5, we obtain the time evolution of the kinetic pressure. As shown in Fig. 6, $P(t)$ decreases monotonically until it reaches a minium value for $t \approx 10 \mu \mathrm{s}$. The time when the minimum pressure is observed coincides with the time for which the plasma size along the line of sight [deduced from the intensity ratio between optically thin and optically thick spectral lines (Sec. 3.4)] is reached.

The $L$-value equals the plasma size deduced from the plume images exposed in Fig. 7. The images illustrate the fast expansion dynamics during a time interval of several tens of nanoseconds, followed by a much slower evolution. Despite a small imperfection that is attributed to the laser energy distribution, the plume is characterized by a well defined expansion front until a time of several hundreds of nanoseconds. At later times, diffusion processes take places, rendering the plume shape symmetric with a leveled intensity distribution.

Although the deduced pressure evolution coincides with the plasma expansion dynamics, the observed minimum value of about one order of magnitude below the ambient gas pressure is questionable. In literature, it is difficult to find information on the properties of laser- 


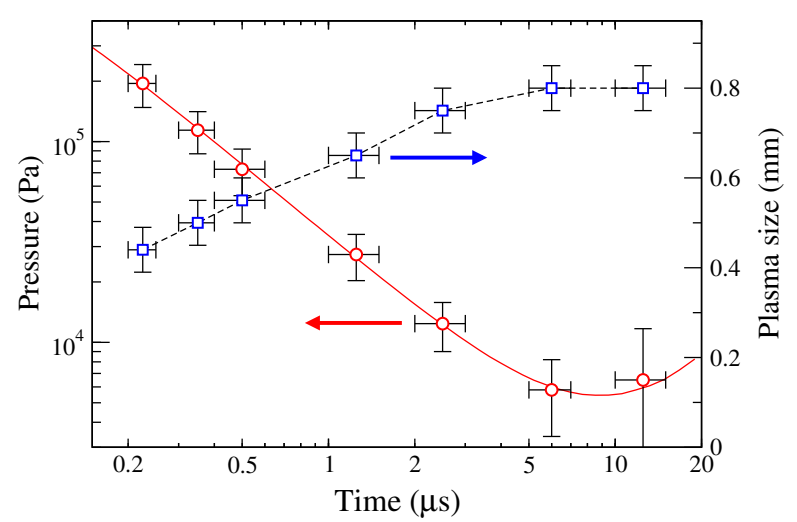

Figure 6: Kinetic pressure (circles) and plasma size (squares) vs time. The pressure evolution is obtained by inserting the time evolution of temperature (Fig. 4) into the $P(T)$-dependence shown in Fig. 5. The vertical error bars represent the measurement uncertainties, whereas the horizontal error bars indicate the time of observation (gate width).

produced plasmas in the late expansion stage. The experimental investigations are challenging. The electron density measurements have large uncertainties as Stark broadening becomes small compared to other line broadening mechanisms. According to the reduced temperature, the observed spectral lines mostly belong to electronic levels of low excitation energy and their emission intensities are affected by self-absorption. Moreover, the plasma runs out of equilibrium as the collisional rates decrease with $n_{e}$ and $T$. Consequently, the late expansion stage of laser-produced plasmas is also challenging for theoretical investigations. The timescale covered by numerical modeling is typically smaller than

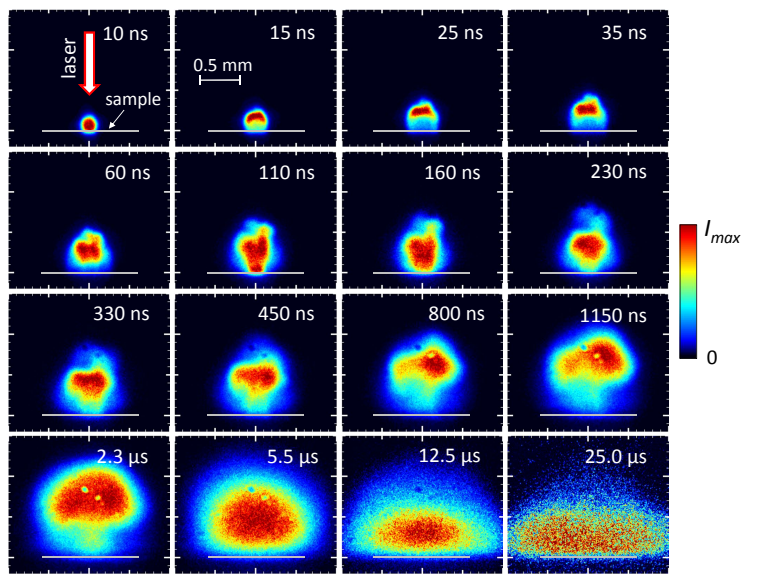

Figure 7: Plasma images recorded for different times. The incoming laser beam and the sample surface location are indicated for $t=10 \mathrm{~ns}$ by the arrow and the horizontal line, respectively. The color scale is adjusted to the maximum intensity for each image. a microsecond [28, 8]. Only Ershov-Pavlov et al. performed calculations on a longer timescale [17]. Considering ablation of aluminum with infrared nanosecond laser pulses in ambient air, the calculations predict a minimum pressure below the atmospheric pressure for a delay of several microseconds, in agreement with our observations.

The pressure decrease below the ambient pressure $\Delta P_{\text {min }}=P_{\text {min }}-P_{a m b}<0$ is characteristic for classical explosions [29]. In that case, the amplitude $\left|\Delta P_{\text {min }}\right|$ is small compared to the ambient pressure $(<10 \%)$. The larger amplitude $\left|\Delta P_{\text {min }}\right|$ in the laser-produced plasma may be explained by the processes of rapid radiative cooling and recombination related to the small plume size.

In a previous work on LIBS analyses of glass [25], the pressure minimum below the ambient gas pressure was not observed. However, due to the presence of oxygen, the plasma was shown to run out of equilibrium for times $t>\mu \mathrm{s}$. This leads to an overestimation of the number densities of oxygen species and consequently to an overvaluation of the kinetic pressure.

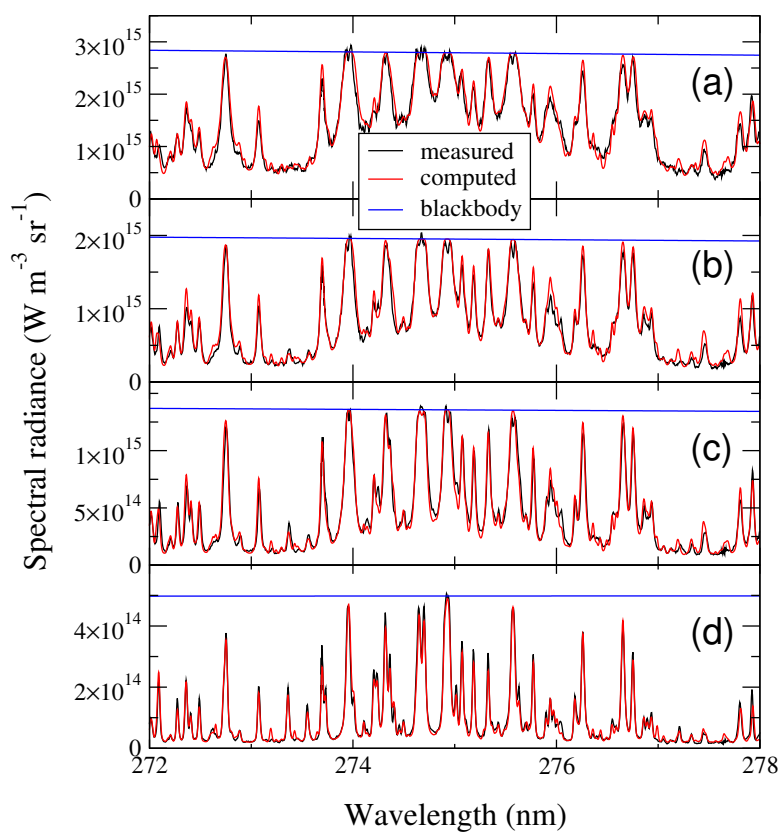

Figure 8: Measured (black lines) and computed (red lines) spectral radiances in the range of strong $\mathrm{Fe}^{+}$-transitions. The recordings were performed for $t=225 \mathrm{~ns}$ (a), $325 \mathrm{~ns}$ (b), $450 \mathrm{~ns}$ (c) and $1250 \mathrm{~ns}$ (d). The blue lines represent the blackbody spectral radiance. 


\subsection{Model validation through spectra simulation}

The measured and computed spectral radiances are displayed in Fig. 8 for a range of several strong $\mathrm{Fe}^{+}$-transitions. More than 300 lines were considered in the calculation. The most intense transitions are listed in Table A.4 (see Appendix A). Among them, several lines are shown to saturate at the same $B_{\lambda}$-value. This behavior is predicted for optically thick lines that are expected to saturate at the blackbody spectral radiance according to Eq. (8). The spectral radiance of these lines is independent of the plasma dimension. Contrarily, the spectral radiance of optically thin transitions increases linearly with $L$ according to Eq. (7). The difference in the $L$-dependence was explored to measure the plasma size along the line of sight. Thus, we adjusted the intensity of the measured spectrum to the computed spectral radiance for the strongest optically thick transitions. Then, we adjusted $L$ so that the measured intensity of optically thin transitions (i.e. Fe I $382.78 \mathrm{~nm}$ ) equals their computed radiance. Applying this procedure to the spectra recorded for $t<1 \mu \mathrm{s}$, we obtain $L$-values in agreement with the plasma diameter deduced from the plume images (see Fig. 7). The plasma emission spectrum is thus well described by the spectral radiance of a uniform plasma in local thermodynamic equilibrium according to Eq. (1).

\section{Conclusion}

The present analyses demonstrate that laser ablation of solid materials in appropriate conditions generates a uniform plasma in local thermodynamic equilibrium. For a clear demonstration, we irradiated stainless steel with UV nanosecond laser pulses under argon background gas. The rich spectrum of iron was explored using both optically thin and optically thick spectral lines. Transitions of negligible optical thickness were used for accurate measurements of temperature, electron density and plasma composition. The knowledge of the plasma properties was then used to calculate the spectral radiance of strongly self-absorbed lines. These lines were shown to saturate at the blackbody spectral radiance in agreement with the radiation model of the uniform LTE plasma. The accurate plasma diagnostics allowed us to deduce the time evolution of the kinetic pressure within the ablated vapor plume. The pressure was shown to decrease monotonically until it reaches a minimum value for a delay of a few microseconds. The minimum pressure was found to be smaller than the ambient gas pressure, in agreement with the computational predictions of Ershov-Pavlov [17]. The precise knowledge of the pressure evolution in the laser-produced ablation plume is of interest for the validation of plasma modeling. It may play the role of a control parameter in material analyses via calibration-free laser-induced breakdown spectroscopy.

\section{Acknowledgements}

The research leading to these results has received funding from LASERLAB-EUROPE (Grant Agreement No. 654148, European Union's Horizon 2020 research and innovation programme). EA and VC acknowledge the financial support from Romanian national projects NUCLEU-LAPLAS and PCCA 226.

\section{Appendix A. spectroscopic data}

The spectroscopic data were taken in priority from the NIST database [30]. For spectral lines for which no

Table A.2: Fe-transitions used for $n_{e}$ and $T$ measurements: wavelength $\lambda$, transition probability $A_{u l}$, energy $E$ and total angular momentum quantum number $J$ of upper (index $u$ ) and lower (index $l$ ) excitation levels, Stark width $w$ and shift $d$ for $n_{e}=1 \times 10^{17} \mathrm{~cm}^{-3}$.

\begin{tabular}{lcrrrrrcc} 
& $\begin{array}{c}\lambda \\
(\mathrm{nm})\end{array}$ & $\begin{array}{r}A_{u l} \\
(\mu \mathrm{s})\end{array}$ & $\begin{array}{r}E_{l} \\
(\mathrm{eV})\end{array}$ & $\begin{array}{c}E_{u} \\
(\mathrm{eV})\end{array}$ & $J_{l}$ & $J_{u}$ & $\begin{array}{c}w \\
(\mathrm{pm})\end{array}$ & $\begin{array}{c}d \\
(\mathrm{pm})\end{array}$ \\
\hline Fe I & 302.064 & 75.90 & 0.00 & 4.10 & 4 & 4 & 4 & 0 \\
Fe I & 322.206 & 86.50 & 2.40 & 6.25 & 5 & 5 & 54 & -10 \\
Fe I & 322.579 & 118.00 & 2.40 & 6.24 & 5 & 6 & 54 & -10 \\
Fe I & 328.675 & 59.90 & 2.18 & 5.95 & 3 & 3 & 20 & 5 \\
Fe I & 344.061 & 17.10 & 0.00 & 3.60 & 4 & 3 & 5 & 2 \\
Fe I & 346.586 & 11.90 & 0.11 & 3.69 & 1 & 1 & 5 & 2 \\
Fe I & 347.545 & 9.75 & 0.09 & 3.65 & 2 & 2 & 5 & 2 \\
Fe I & 349.057 & 6.14 & 0.05 & 3.60 & 3 & 3 & 5 & 2 \\
Fe I & 353.656 & 99.50 & 2.88 & 6.38 & 2 & 3 & 60 & 0 \\
Fe I & 354.108 & 86.50 & 2.85 & 6.35 & 4 & 5 & 60 & 0 \\
Fe I & 354.208 & 95.10 & 2.87 & 6.36 & 3 & 4 & 60 & 0 \\
Fe I & 355.492 & 140.00 & 2.83 & 6.32 & 5 & 6 & 60 & 0 \\
Fe I & 360.668 & 82.90 & 2.69 & 6.13 & 5 & 6 & 15 & 3 \\
Fe I & 365.147 & 58.30 & 2.76 & 6.15 & 3 & 4 & 10 & 3 \\
Fe I & 370.557 & 3.21 & 0.05 & 3.40 & 3 & 3 & 5 & 0 \\
Fe I & 371.993 & 16.20 & 0.00 & 3.33 & 4 & 5 & 5 & 0 \\
Fe I & 372.256 & 4.97 & 0.09 & 3.42 & 2 & 2 & 5 & 0 \\
Fe I & 373.332 & 6.48 & 0.11 & 3.43 & 1 & 1 & 5 & 0 \\
Fe I & 373.486 & 90.10 & 0.86 & 4.18 & 5 & 5 & 6 & 0 \\
Fe I & 373.713 & 14.10 & 0.05 & 3.37 & 3 & 4 & 7 & 0 \\
Fe I & 374.556 & 11.50 & 0.09 & 3.40 & 2 & 3 & 7 & 0 \\
Fe I & 374.826 & 9.15 & 0.11 & 3.42 & 1 & 2 & 7 & 0 \\
Fe I & 374.948 & 76.30 & 0.91 & 4.22 & 4 & 4 & 8 & 0 \\
Fe I & 375.823 & 63.40 & 0.96 & 4.26 & 3 & 3 & 8 & 0 \\
Fe I & 376.554 & 95.10 & 3.24 & 6.53 & 6 & 7 & 11 & 2 \\
Fe I & 381.584 & 112.00 & 1.48 & 4.73 & 4 & 3 & 13 & 5 \\
Fe I & 382.042 & 66.70 & 0.86 & 4.10 & 5 & 4 & 9 & 3 \\
Fe I & 382.430 & 38.07 & 3.30 & 6.54 & 4 & 4 & 8 & 0 \\
Fe I & 382.444 & 2.83 & 0.00 & 3.24 & 4 & 3 & 6 & 0 \\
\hline & & & & & & & &
\end{tabular}


Table A.3: Additional Fe-transitions used for $n_{e}$ and $T$ measurements: wavelength $\lambda$, transition probability $A_{u l}$, energy $E$ and total angular momentum quantum number $J$ of upper (index $u$ ) and lower (index $l$ )

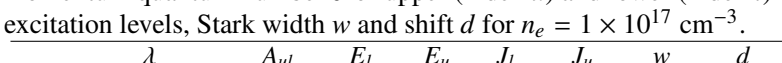

\begin{tabular}{|c|c|c|c|c|c|c|c|c|}
\hline & $(\mathrm{nm})$ & $\begin{array}{l}A_{u l} \\
(\mu \mathrm{s})\end{array}$ & $\begin{array}{r}L_{l} \\
(\mathrm{eV})\end{array}$ & $\begin{array}{r}\boldsymbol{L}_{u} \\
(\mathrm{eV})\end{array}$ & 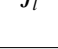 & . & $(\mathrm{pm})$ & $\begin{array}{c}a \\
(\mathrm{pm})\end{array}$ \\
\hline Fe I & 382.588 & 59.70 & 0.91 & 4.15 & 4 & 3 & 9 & 3 \\
\hline $\mathrm{Fe} \mathrm{I}$ & 382.782 & 105.00 & 1.56 & 4.80 & 3 & 2 & 13 & 5 \\
\hline $\mathrm{Fe} \mathrm{I}$ & 385.637 & 4.64 & 0.05 & 3.27 & 3 & 2 & 6 & 0 \\
\hline Fe I & 385.991 & 9.69 & 0.00 & 3.21 & 4 & 4 & 6 & 0 \\
\hline $\mathrm{Fe} \mathrm{I}$ & 387.857 & 6.17 & 0.09 & 3.28 & 2 & 1 & 6 & 0 \\
\hline $\mathrm{Fe} \mathrm{I}$ & 388.628 & 5.29 & 0.05 & 3.24 & 3 & 3 & 6 & 0 \\
\hline Fe I & 388.705 & 3.52 & 0.91 & 4.10 & 4 & 4 & 9 & 3 \\
\hline $\mathrm{Fe} \mathrm{I}$ & 389.566 & 9.39 & 0.11 & 3.29 & 1 & 0 & 6 & 0 \\
\hline $\mathrm{Fe} \mathrm{I}$ & 392.291 & 1.08 & 0.05 & 3.21 & 3 & 4 & 6 & 0 \\
\hline $\mathrm{Fe} \mathrm{I}$ & 404.581 & 86.20 & 1.48 & 4.55 & 4 & 4 & 15 & 3 \\
\hline $\mathrm{Fe} \mathrm{I}$ & 419.909 & 49.20 & 3.05 & 6.00 & 4 & 5 & 12 & 2 \\
\hline $\mathrm{Fe} \mathrm{I}$ & 420.203 & 8.22 & 1.48 & 4.43 & 4 & 4 & 12 & 3 \\
\hline $\mathrm{Fe} \mathrm{I}$ & 422.743 & 52.90 & 3.33 & 6.26 & 5 & 6 & 100 & 25 \\
\hline $\mathrm{Fe} \mathrm{I}$ & 425.079 & 10.20 & 1.56 & 4.47 & 3 & 3 & 20 & 0 \\
\hline $\mathrm{Fe} \mathrm{I}$ & 426.047 & 39.90 & 2.40 & 5.31 & 5 & 5 & 50 & 20 \\
\hline $\mathrm{Fe} \mathrm{I}$ & 438.354 & 50.00 & 1.48 & 4.31 & 4 & 5 & 15 & 3 \\
\hline $\mathrm{Fe} \mathrm{I}$ & 441.512 & 11.90 & 1.61 & 4.42 & 2 & 3 & 15 & 3 \\
\hline Fe I & 492.050 & 35.80 & 2.83 & 5.35 & 5 & 4 & 40 & 20 \\
\hline Fe I & 526.954 & 1.27 & 0.86 & 3.21 & 5 & 4 & 7 & 2 \\
\hline $\mathrm{Fe} \mathrm{I}$ & 538.337 & 78.10 & 4.31 & 6.61 & 5 & 6 & 185 & -21 \\
\hline $\mathrm{Fe} \mathrm{I}$ & 538.948 & 12.76 & 4.42 & 6.72 & 3 & 3 & 150 & 30 \\
\hline $\mathrm{Fe} \mathrm{I}$ & 539.146 & 6.86 & 4.15 & 6.45 & 3 & 2 & 30 & 10 \\
\hline Fe II & 292.659 & 5.10 & 0.99 & 5.22 & $7 / 2$ & $9 / 2$ & 6 & 0 \\
\hline Fe II & 294.440 & 35.00 & 1.70 & 5.90 & $3 / 2$ & $1 / 2$ & 8 & 4 \\
\hline Fe II & 294.765 & 20.10 & 1.67 & 5.88 & $5 / 2$ & $3 / 2$ & 8 & 4 \\
\hline Fe II & 298.483 & 43.00 & 1.67 & 5.82 & $5 / 2$ & $5 / 2$ & 13 & 4 \\
\hline Fe II & 300.264 & 17.90 & 1.70 & 5.82 & $3 / 2$ & $5 / 2$ & 8 & 4 \\
\hline Fe II & 307.717 & 14.00 & 4.08 & 8.10 & $13 / 2$ & $11 / 2$ & 13 & 0.0 \\
\hline Fe II & 315.420 & 20.60 & 3.77 & 7.70 & $9 / 2$ & $9 / 2$ & 7 & -2 \\
\hline Fe II & 316.785 & 16.00 & 3.81 & 7.73 & $7 / 2$ & $7 / 2$ & 7 & -2 \\
\hline Fe II & 317.753 & 17.40 & 3.90 & 7.80 & $7 / 2$ & $7 / 2$ & 7 & -2 \\
\hline Fe II & 322.774 & 8.90 & 1.67 & 5.51 & $5 / 2$ & $7 / 2$ & 15 & 0 \\
\hline
\end{tabular}

transition probability was given by NIST, the $A_{u l}$-values were taken from the Kurucz database [31].

[1] D. W. Hahn, N. Omenetto, Laser-induced breakdown spectroscopy (LIBS), Part I: Review of basic diagnostics and plasmaparticle interactions: still-challenging issues within the analytical plasma community, Appl. Spectrosc. 64 (2010) 335A-366A

[2] G. Cristoforetti, E. Tognoni, L. A. Gizzi, Thermodynamic equilibrium states in laser-induced plasmas: From the general case to laser-induced breakdown spectroscopy plasmas, Spectrochim. Acta Part B: Atom. Spectrosc. 90 (2013) 1-22.

[3] I. N. Mihailescu, J. Hermann, Laser-plasma interactions, in: P. Schaaf (Ed.), Laser Processing of Materials, Springer, Berlin, 2010, pp. 49-88.

[4] J. Hermann, A. Lorusso, A. Perrone, F. Strafella, C. Dutouquet, B. Torralba, Simulation of emission spectra from nonuniform reactive laser-induced plasmas, Phys. Rev. E 92 (2015) 053103 $1-15$.

[5] J. Hermann, C. Gerhard, E. Axente, C. Dutouquet, Comparative investigation of laser ablation plumes in air and argon by analysis of spectral line shapes: Insights on calibration-free laserinduced breakdown spectroscopy, Spectrochim. Acta Part B: Atom. Spectrosc. (2014) 189-196.

[6] J. Hermann, D. Grojo, E. Axente, C. Gerhard, M. Burger, V. Craciun, Ideal radiation source for plasma spectroscopy generated by laser ablation, Phys. Rev. E 96 (2017) 053210 1-6.
Table A.4: Most intense transitions in the range from 272 to $278 \mathrm{~nm}$ : wavelength $\lambda$, transition probability $A_{u l}$, energy $E$ and total angular momentum quantum number $J$ of upper (index $u$ ) and lower (index $l$ ) excitation levels, Stark width $w$ and shift $d$ for $n_{e}=1 \times 10^{17} \mathrm{~cm}^{-3}$.

\begin{tabular}{lcrrccccc} 
& $\begin{array}{c}\lambda \\
(\mathrm{nm})\end{array}$ & $\begin{array}{r}A_{u l} \\
(\mu \mathrm{s})\end{array}$ & $\begin{array}{r}E_{l} \\
(\mathrm{eV})\end{array}$ & $\begin{array}{c}E_{u} \\
(\mathrm{eV})\end{array}$ & $J_{l}$ & $J_{u}$ & $\begin{array}{c}w \\
(\mathrm{pm})\end{array}$ & $\begin{array}{c}d \\
(\mathrm{pm})\end{array}$ \\
\hline Fe II & 272.754 & $93.80^{a}$ & 1.04 & 5.58 & $5 / 2$ & $3 / 2$ & 7 & 0 \\
Fe II & 273.697 & $122.00^{b}$ & 1.08 & 5.60 & $3 / 2$ & $1 / 2$ & 4 & 0 \\
Fe II & 273.955 & $221.00^{a}$ & 0.99 & 5.51 & $7 / 2$ & $7 / 2$ & 6 & 0 \\
Fe II & 274.320 & $197.00^{b}$ & 1.10 & 5.62 & $1 / 2$ & $3 / 2$ & 6 & 0 \\
Cr II & 274.364 & $63.12^{c}$ & 1.48 & 6.00 & $1 / 2$ & $3 / 2$ & 4 & 0 \\
Fe II & 274.648 & $205.00^{b}$ & 1.08 & 5.59 & $3 / 2$ & $5 / 2$ & 6 & 0 \\
Fe II & 274.698 & $169.00^{d}$ & 1.04 & 5.55 & $5 / 2$ & $5 / 2$ & 5 & 0 \\
Cr II & 274.898 & $109.30^{c}$ & 1.49 & 6.00 & $3 / 2$ & $3 / 2$ & 4 & 0 \\
Fe II & 274.918 & $121.00^{a}$ & 1.08 & 5.58 & $3 / 2$ & $3 / 2$ & 8 & 0 \\
Fe II & 274.932 & $216.00^{b}$ & 1.04 & 5.55 & $5 / 2$ & $7 / 2$ & 5 & 0 \\
Fe II & 274.949 & $116.00^{b}$ & 1.10 & 5.60 & $1 / 2$ & $1 / 2$ & 8 & 0 \\
Cr II & 275.073 & $87.26^{c}$ & 1.51 & 6.01 & $5 / 2$ & $5 / 2$ & 4 & 0 \\
Cr II & 275.187 & $49.26^{c}$ & 1.53 & 6.03 & $7 / 2$ & $7 / 2$ & 4 & 0 \\
Fe II & 275.329 & $189.00^{e}$ & 3.27 & 7.77 & $9 / 2$ & $11 / 2$ & 5 & 0 \\
Fe II & 275.574 & $215.00^{a}$ & 0.99 & 5.48 & $7 / 2$ & $9 / 2$ & 5 & 0 \\
Cr II & 275.772 & $98.11^{c}$ & 1.51 & 6.00 & $5 / 2$ & $3 / 2$ & 4 & 0 \\
Cr II & 276.259 & $162.60^{c}$ & 1.53 & 6.01 & $7 / 2$ & $5 / 2$ & 4 & 0 \\
Cr II & 276.654 & $222.30^{c}$ & 1.55 & 6.03 & $9 / 2$ & $7 / 2$ & 4 & 0 \\
Fe II & 276.750 & $158.00^{f}$ & 3.24 & 7.72 & $11 / 2$ & $13 / 2$ & 5 & 0 \\
Fe II & 277.930 & $100.00^{f}$ & 3.27 & 7.73 & $9 / 2$ & $7 / 2$ & 6 & 0 \\
\hline
\end{tabular}

[7] I. B. Gornushkin, A. Y. Kazakov, Kinetic model of stimulated emission created by resonance pumping of aluminum laserinduced plasma, J. Appl. Phys. 121 (2017) 213303 1-11.

[8] S. V. Shabanov, I. B. Gornushkin, Two-dimensional axisymmetric models of laser induced plasmas relevant to laser induced breakdown spectroscopy, Spectrochim. Acta Part B: Atom. Spectrosc. 100 (2014) 147-172.

[9] C. Aragón, J. A. Aguilera, Characterization of laser induced plasmas by optical emission spectroscopy: A review of experiments and methods, Spectrochim. Acta Part B: Atom. Spectrosc. 63 (2008) 893-916.

[10] K. K. Herrera, E. Tognoni, N. Omenetto, B. W. Smith, J. D. Winefordner, Semi-quantitative analysis of metal alloys, brass and soil samples by calibration-free laser-induced breakdown spectroscopy: recent results and considerations, J. Anal. At. Spectrom. 24 (2009) 413-425.

[11] M. Burger, J. Hermann, Stark broadening measurements in plasmas produced by laser ablation of hydrogen containing compounds, Spectrochim. Acta Part B: Atom. Spectrosc. 122 (2016) $118-126$.

[12] M. Ivković, N. Konjević, Stark width and shift for electron number density diagnostics of low temperature plasma: Application to silicon LIBS, Spectrochim. Acta Part B: Atom. Spectrosc. 131 (2017) 79-92.

[13] G. Cristoforetti, G. Lorenzetti, S. Legnaioli, V. Palleschi, Investigation on the role of air in the dynamical evolution and thermodynamic state of a laser-induced aluminium plasma by spatial- and time-resolved spectroscopy, Spectrochim. Acta Part B: Atom. Spectrosc. 65 (2010) 787-796.

[14] A. D. Giacomo, J. Hermann, Laser-induced plasma emission: from atomic to molecular spectra, J. Phys. D: Appl. Phys. 50 (2017) 183002 1-17.

[15] Z. Y. Chen, A. Bogaerts, Laser ablation of cu and plume expansion into 1 atm ambient gas, J. Appl. Phys. 97 (2005) 063305 $1-11$.

[16] G. Clair, D. L'Hermite, 1D modelling of nanosecond laser ablation of copper samples in argon at $\mathrm{P}=1$ atm with a wavelength 
of 532 nm, J. Appl. Phys. 110 (2011) 083307 1-10.

[17] E. A. Ershov-Pavlov, K. Y. Katsalap, K. L. Stepanov, U. A Stankevich, Time-space distribution of laser-induced plasma parameters and its influence on emission spectra of the laser plumes, Spectrochim. Acta Part B: Atom. Spectrosc. 63 (2008) 1024-1037.

[18] J. Cooper, Plasma spectroscopy, Rep. Prog. Phys. 29 (1966) 35130.

[19] H. R. Griem, Plasma spectroscopy, Academic, New York, 1964

[20] X. Z. Zhao, L. J. Shen, T. X. Lu, K. Niemax, Spatial distributions of electron-density in microplasmas produced by laser ablation of solids, Appl. Phys. B: Photophys. Laser Chem. 55 (1992) 327-330.

[21] E. Tognoni, V. Palleschi, M. Corsi, G. Cristoforetti, N. Omenetto, I. Gornushkin, B. W. Smith, J. D. Winefordner, From sample to signal in laser-induced breakdown spectroscopy: a complex route to quantitative analysis, in: A. W. Miziolek, V. Palleschi, I. Schechter (Eds.), Laser-induced breakdown spectroscopy, Cambridge University, Berlin, 2006, pp 122-194.

[22] S. Zielinska, S. Pellerin, K. Dzierzega, F. Valensi, K. Musiol, F. Briand, Measurement of atomic Stark parameters of many Mn I and Fe I spectral lines using GMAW process, J. Phys. D: Appl. Phys. 43 (2010) 434005 1-10

[23] E. Axente, J. Hermann, G. Socol, L. Mercadier, S. A. Beldjilali, M. Cirisan, C. R. Luculescu, C. Ristoscu, I. N. Mihailescu, V. Craciun, Accurate analysis of indium-zinc oxide thin films via laser-induced breakdown spectroscopy based on plasma modeling, J. Anal. At. Spectrom. 29 (2014) 553-564.

[24] J. Hermann, C. Dutouquet, Local thermal equilibrium plasma modeling for analyses of gas-phase reactions during reactivelaser ablation, J. Appl. Phys. 91 (2002) 10188-10193.

[25] C. Gerhard, J. Hermann, L. Mercadier, L. Loewenthal, E. Axente, C. R. Luculescu, T. Sarnet, M. Sentis, W. Viöl, Quantitative analyses of glass via laser-induced breakdown spectroscopy in argon, Spectrochim. Acta Part B: Atom. Spectrosc. 101 (2014) 32-45.

[26] N. Konjević, A. Lesage, J. R. Führ, W. L. Wiese, Experimental Stark widths and shifts for spectral lines of neutral and ionized atoms (A critical review of selected data for the period 1989 through 2000), J. Phys. Chem. Ref. Data 31 (2002) 819-921.

[27] A. W. Miziolek, V. Palleschi, I. Schechter (Eds.), Laser-induced breakdown spectroscopy, Cambridge University, Berlin, 2006.

[28] S. S. Harilal, G. V. Miloshevsky, P. K. Diwakar, N. L. LaHaye, A. Hassanein, Experimental and computational study of complex shockwave dynamics in laser ablation plumes in argon atmosphere, Phys. Plasmas 19 (2012) 083504 1-11.

[29] M. D. Goel, V. A. Matsagar, A. K. Gupta, S. Marburg, An abridged review of blast wave parameters, Defense Sci. J. 62 (5) (2012) 300-306.

[30] A. Kramida, Y. Ralchenko, J. Reader, NIST Atomic Spectra Database (version 5.3), National Institute of Standards and Technology, Gaithersburg, MD (2015). URL http: //physics.nist.gov/asd

[31] P. L. Smith, C. Heise, J. R. Esmond, R. L. Kurucz, Atomic spectral line database built from atomic data files from R. L. Kurucz CD-ROM 23 (2011).

URL http://www.pmp.uni-hannover.de/cgi-bin/ssi/test/kurucz/sekur.html 